\title{
Electrical response of molecular chains in density functional theory: Ultranonlocal response from a semilocal functional
}

\author{
Rickard Armiento, Stephan Kümmel and Thomas Körzdörfer
}

\section{Post Print}

N.B.: When citing this work, cite the original article.

Original Publication:

Rickard Armiento, Stephan Kümmel and Thomas Körzdörfer, Electrical response of molecular chains in density functional theory: Ultranonlocal response from a semilocal functional, 2008, Physical Review B. Condensed Matter and Materials Physics, (77), 16, 165106.

http://dx.doi.org/10.1103/PhysRevB.77.165106

Copyright: American Physical Society http://www.aps.org/

Postprint available at: Linköping University Electronic Press http://urn.kb.se/resolve?urn=urn:nbn:se:liu:diva-86295 


\title{
Electrical response of molecular chains in density functional theory: Ultranonlocal response from a semilocal functional
}

\author{
R. Armiento, S. Kümmel, and T. Körzdörfer \\ Theoretische Physik, Universität Bayreuth, D-95440 Bayreuth, Germany
}

(Received 28 September 2007; published 2 April 2008)

\begin{abstract}
An exchange potential functional is constructed from semi-local quantities and is shown to reproduce hydrogen chain polarizabilities with the same accuracy as exact exchange methods. We discuss the exchange potential features that are essential for accurate polarizability calculations, i.e., derivative discontinuities and the potential step structure. The possibility of a future generalization of the methods into a complete semi-local exchange-correlation functional is discussed.
\end{abstract}

DOI: 10.1103/PhysRevB.77.165106

\section{INTRODUCTION}

Molecular chains can have a large linear and nonlinear electrical response, while being both cheap to produce and easy to process. This makes them interesting for industrial use for, e.g., nonlinear optics devices. ${ }^{1}$ However, no largescale computationally feasible electronic structure theory currently gives an all around good description of the properties of these systems.

Generally speaking, structural properties are described well by density functional theory methods (DFT) ${ }^{2,3}$ with a local or semi-local exchange-correlation (xc) functional, such as the local density approximation (LDA) ${ }^{3}$ or a generalized gradient approximation (GGA). ${ }^{4,5}$ However, typical semi-local DFT methods are unable to describe the polarizabilities of molecular chains well ${ }^{6-9}$ and make orders of magnitude errors for the electrical response of, e.g., polyacetylene.

On the other hand, one can obtain accurate polarizabilities by turning to DFT with completely non-local exact exchange $^{10-13}$ or self-interaction corrections. ${ }^{14-16}$ However, bare exact exchange does not describe structural properties as accurately as semi-local methods, and for such applications, it has to be combined with some correlation treatment. Another problem with both exact exchange and selfinteraction correction methods is that they are computationally more demanding than semi-local methods, which hinders calculations on larger systems. It is therefore of great interest for a successful theoretical description of molecular chains to find a way to adjust current semi-local methods to include the necessary features from the exact exchange necessary for accurate polarizability calculations. This is the topic of the present paper.

Previous works have pointed out specific exchange potential features as crucial for describing the polarizabilities of molecular chains. ${ }^{7,10,12}$ It is by no means a priori evident that these features can be successfully included in a semi-local description; they could be too closely linked to the non-local nature of exact exchange. Nonetheless, the present paper presents an exchange potential functional from semi-local methods that reproduces these features for hydrogen chain test systems and gives polarizabilities that are of the same accuracy as those from exact exchange methods. It is an important result in itself that such a functional even exists, but the functional also opens for the opportunity to combine the present methods with a correlation treatment for accomplishing the goal of a good description of both structural and electrical properties of, e.g., molecular chains.

In the following, we first discuss the exchange potential features that are essential for accurate polarizability calculations of molecular chains, i.e., derivative discontinuities and the related step structure in the exchange potential. These features are shown to be present in an exchange potential correction term recently proposed by Becke-Johnson ${ }^{17}$ (BJ). However, the term is observed to have deficiencies in calculations with an external electrical field. Therefore, we further develop this correction to resolve the problems. An important aspect of our work is that the functional is developed directly for the potential, in contrast to the usual approach of developing functionals for the energy. We show that numerical results for the polarizabilities from the fully corrected exchange potential functional are as accurate as those obtained from exact exchange methods.

\section{EXCHANGE FEATURES ESSENTIAL FOR THE ELECTRICAL RESPONSE}

The designation "the derivative discontinuity" in DFT is often taken to refer to the discontinuous jumps in the derivative of the total energy with respect to particle number, $d E / d N$. This has been thoroughly discussed by, e.g., Perdew et al. ${ }^{18}$ and a quick summary is given in the following. Consider two different neutral atoms that are well separated. If the chemical potential $\mu=d E / d N$ is continuous for both individual atoms, a fractional electron transfer in the direction of the higher chemical potential would decrease the total energy and lead to an incorrect ground state with non-neutral atoms. Hence, there must be a discontinuous step in $d E / d N$ at integer electron number $N$. In the Kohn-Sham $(\mathrm{KS})^{3}$ scheme, the discontinuous step in the total energy derivative relates directly to corresponding steps in the derivatives of the noninteracting kinetic energy $T_{s}$ and the xc energy $E_{\mathrm{xc}}{ }^{36}$

Note that discontinuous steps appear in $d E / d N$ even for ordinary semi-local approximations of $E_{\mathrm{xc}}$ such as LDA. Figure 1 shows the total energy $E$ with an inset for $E_{\mathrm{x}}$ for a fully self-consistent LDA exchange-only DFT calculation of a $\mathrm{Mg}$ ion with different (fractional) electron occupation $N$. There are clearly visible kinks in the curve for the integer particle numbers where new shells are opened. The origin of the discontinuities can be illustrated by looking at the derivatives 


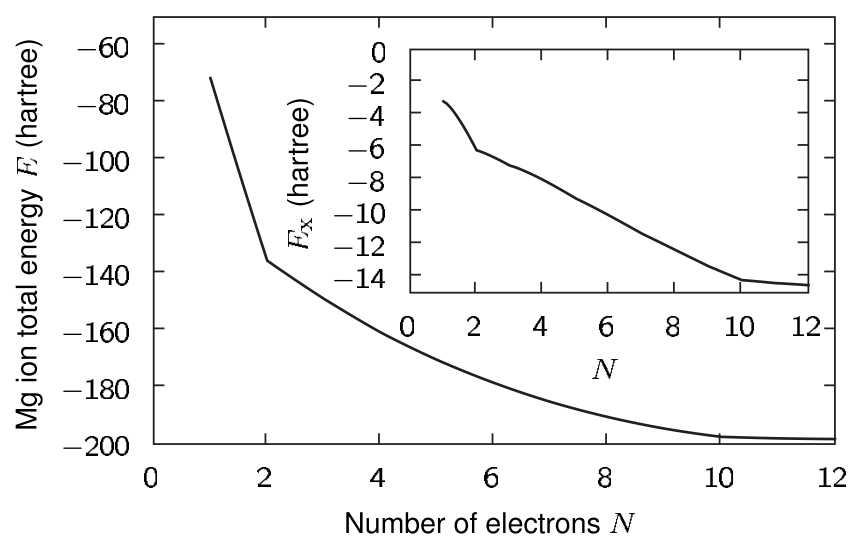

FIG. 1. Total and exchange energy of a $\mathrm{Mg}$ ion versus electron occupation number, calculated with the fully self-consistent exchange-only LDA. New electron shells are started when $N$ is equal to 2 and 10 . The figure shows that even for exchange-only LDA, both $d E / d N$ and $d E_{\mathrm{x}} / d N$ are discontinuous at these points.

for a simple two KS orbital system, $\phi_{1}(\mathbf{r}), \phi_{2}(\mathbf{r})$,

$$
\begin{gathered}
\left.\frac{d E_{\mathrm{x}}^{\mathrm{LDA}}}{d N}\right|_{N=1-\omega} \sim \int\left[(1-\omega)\left|\phi_{1}\right|^{2}\right]^{1 / 3}\left|\phi_{1}\right|^{2} d^{3} \mathbf{r}, \\
\left.\frac{d E_{\mathrm{x}}^{\mathrm{LDA}}}{d N}\right|_{N=1+\omega} \sim \int\left(\left|\phi_{1}\right|^{2}+\omega\left|\phi_{2}\right|^{2}\right)^{1 / 3}\left|\phi_{2}\right|^{2} d^{3} \mathbf{r} .
\end{gathered}
$$

The only way to avoid a discontinuous step in the $\omega \rightarrow 0$ limit is thus if

$$
\int\left|\phi_{1}\right|^{8 / 3} d^{3} \mathbf{r}=\int\left|\phi_{1}\right|^{2 / 3}\left|\phi_{2}\right|^{2} d^{3} \mathbf{r},
$$

and this is not generally true (e.g., for hydrogen orbitals from different shells). However, note that, in contrast, the LDA exchange potential $v_{\mathrm{x}}=\delta E_{\mathrm{x}} / \delta n(\mathbf{r})$ has no discontinuous shift in the same limit. For example, in the simple two orbital system,

$$
\begin{aligned}
& \left.\frac{\delta E_{\mathrm{x}}^{\mathrm{LDA}}}{\delta n(\mathbf{r})}\right|_{N=1-\omega} \sim\left[(1-\omega)\left|\phi_{1}\right|^{2}\right]^{1 / 3}, \\
& \left.\frac{\delta E_{\mathrm{x}}^{\mathrm{LDA}}}{\delta n(\mathbf{r})}\right|_{N=1+\omega} \sim\left(\left|\phi_{1}\right|^{2}+\omega\left|\phi_{2}\right|^{2}\right)^{1 / 3} .
\end{aligned}
$$

Hence, one can make the distinction that while the LDA exchange energy has a derivative discontinuity with respect to particle number at certain integers, the exchange potential has no such discontinuity. The same holds true, as far as the authors know, for other accepted semi-local methods. However, several works have discussed that the true exact exchange functional has a potential discontinuity ${ }^{19-21}$ For example, Krieger, $\mathrm{Li}$, and Iafrate $(\mathrm{KLI})^{20}$ observed that with just a tiny fraction of occupancy in a new shell (they went as low as $10^{-15}$ ), the exchange potential is shifted discontinuously.
The discontinuous shift in the exchange potential turns out to be closely related to the "step structure" of the exchange potential of atoms, as discussed by, e.g., van Leeuwen et $a .^{22} \mathrm{~A}$ bounded system, like an atom or a molecule, has a shell structure with lower energy KS orbitals dominating inner shells and higher orbitals consecutively dominating outer shells. In the spatial region between shells, the exact exchange potential changes rapidly and makes a distinct "step." Such a step is present regardless of how small the occupancy of a shell is, as long as it is greater than zero. Hence, in the fractionally filled orbital case, the orbitals are filled with a successively increasing fractional particle number and a new step is created at the exact point when a new shell is opened. If a boundary condition $v_{\mathrm{x}} \rightarrow 0$ as $\mathbf{r} \rightarrow \infty$ is enforced for the potential, it shifts the whole potential discontinuously (just as was observed by KLI). Similar considerations also apply for the time-dependent extension of DFT. $^{23,24}$

\section{BECKE-JOHNSON TERM}

A relevant question at this point is as follows: Can an expression based on semi-local quantities still reproduce the step structure and derivative discontinuity in the exchange potential at integer particle numbers? BJ have recently presented an expression with such properties. ${ }^{17}$ They proposed an exchange potential functional starting from the (nonlocal) Slater exchange, (hartree atomic units $\hbar=e_{\mathrm{c}}=m_{e}=1$ are used here and throughout),

$$
v_{\mathrm{x} \sigma}^{\text {Slater }}(\mathbf{r})=-\frac{1}{n_{\sigma}} \int \frac{\left|\sum_{i} m_{i \sigma} \phi_{i \sigma}^{*}(\mathbf{r}) \phi_{i \sigma}\left(\mathbf{r}^{\prime}\right)\right|^{2}}{\left|\mathbf{r}-\mathbf{r}^{\prime}\right|} d^{3} \mathbf{r}^{\prime},
$$

where $\phi_{i \sigma}$ is the $i$ th KS orbital of spin $\sigma$ (up or down) with occupation $m_{i \sigma}$, making the total occupancy $N=\Sigma_{\sigma i} m_{i \sigma}$, and $n_{\sigma}$ is the electron spin density

$$
n_{\sigma}=\sum_{i} m_{i \sigma}\left|\phi_{i \sigma}\right|^{2} .
$$

The Slater exchange has no potential step structure in itself. However, BJ added a semi-local correction term,

$$
v_{\mathrm{x} \sigma}^{\mathrm{BJ}}=C_{\Delta v} \sqrt{\frac{2 \tau_{\sigma}}{n_{\sigma}}}, \quad C_{\Delta v}=\frac{1}{\pi} \sqrt{\frac{5}{12}},
$$

where $\tau_{\sigma}$ is the kinetic energy spin density,

$$
\tau_{\sigma}=\frac{1}{2} \sum_{i} m_{i \sigma}\left|\nabla \phi_{i \sigma}\right|^{2}
$$

The BJ paper demonstrated that for a set of atoms, the correction term introduces a step structure very similar to that of the exact exchange.

In addition to the findings of $\mathrm{BJ}$, we have found that the steps introduced by the correction behave properly in the context of fractionally occupied orbitals, as illustrated in Fig. 2. This means that not only does the correction term give a step structure in the exchange potential similar to the correct one, but this step structure is related to the derivative discon- 


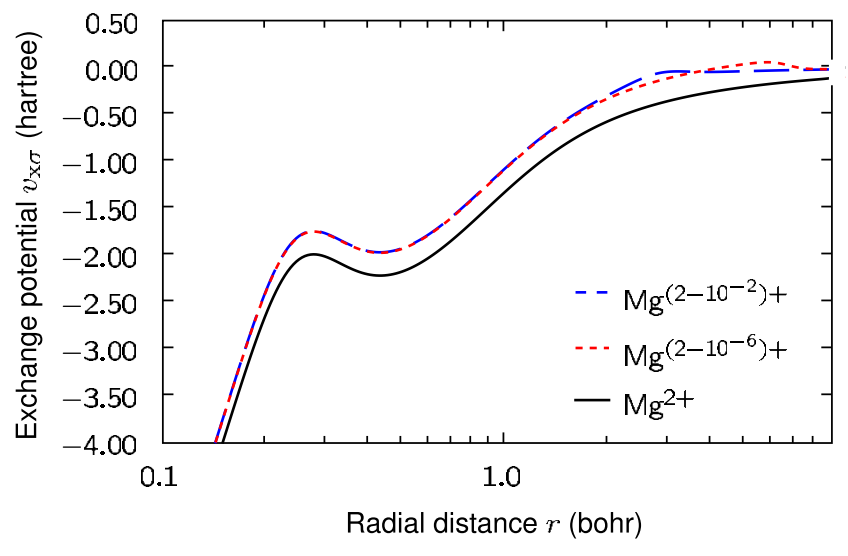

FIG. 2. (Color online) The exchange potential calculated with Slater exchange plus the BJ correction term for fully doubly ionized magnesium atom $\mathrm{Mg}^{2+}$ (fully drawn black line) and the same system but with small fractions of an electron added, $10^{-2}$ (dotted red) and $10^{-6}$ (dashed blue). The last two lines are virtually indistinguishable, which means that the BJ correction term makes the graph discontinuously shifted just as the exact exchange does-precisely when a new orbital shell is opened. The detailed shape of the shift may not be absolutely accurate compared to an exact exchange calculation (not shown, compare to Ref. 20, Fig. 10), but the fact that such a shift is at all present makes for an important difference to other semi-local methods. Also note that the step moves to larger values of $r$ with decreasing occupation number, as it should.

tinuity in a way surprisingly similar to exact exchange. The potential is shifted discontinuously precisely at those integer particle numbers that open a new shell. In the following, we will investigate how the correction term accomplishes this feat.

\section{ASYMPTOTICS OF THE BECKE-JOHNSON TERM WHEN NO ELECTRICAL FIELD IS PRESENT}

The original BJ paper offers little discussion on how the simple form of Eq. (8) achieves the steps in the potential traditionally associated with non-local exchange methods. Some insight is given by a study of the asymptotics of the expression. For simplicity, consider a one-dimensional system with KS orbitals $\phi_{i \sigma}(z)$ and corresponding energies $\epsilon_{i \sigma}$. The highest occupied orbital index is $I$ (where $I=N / 2$ if the system is in its ground state and $N$ is even), and the energy zero is chosen so that all bound states have negative energy, $\epsilon_{i \sigma} \leqslant 0$ for $i \leqslant I$. In the large $z$ asymptotic of a bounded system, the orbitals fall off exponentially as $\phi_{i \sigma}(z) \rightarrow$ $\sim e^{-\sqrt{-2 \epsilon_{i \sigma}} z}$ (cf. Ref. 25). In this limit, the magnitude of the highest occupied orbital will eventually become exponentially larger than the magnitude of lower orbitals. The sums over all occupied orbitals in the electron spin density and kinetic energy spin density expressions, Eqs. (7) and (9), become completely dominated by the term for the highest occupied orbital. Hence,

$$
v_{\mathrm{x} \sigma}^{\mathrm{BJ}} \rightarrow C_{\Delta v}\left|\frac{\nabla \phi_{I \sigma}(z)}{\phi_{I \sigma}(z)}\right|=C_{\Delta v} \sqrt{-2 \epsilon_{I \sigma}} .
$$

Surprisingly, this expression is completely independent of the occupation numbers, $m_{i \sigma}$. Hence, if we start from a con- figuration where the highest occupied orbital is $I_{0}$ and then add some fractional occupancy $m$ to open up the next higher orbital, the asymptotic of the BJ correction term is shifted by

$$
C_{\Delta v}\left(\sqrt{-2 \epsilon_{\left(I_{0}+1\right) \sigma}}-\sqrt{-2 \epsilon_{I_{0} \sigma}}\right) .
$$

This shift is constant, regardless of the actual value of the added occupancy $m$, until it becomes large enough to open yet another higher orbital.

Another useful observation from the above derivation is that the boundary condition $v_{x} \rightarrow 0$ as $z \rightarrow \infty$ can now be easily enforced by subtracting the found asymptotic term. If this is done, the complete correction to Slater exchange becomes

$$
v_{\mathrm{x} \sigma}^{\mathrm{corr}}=C_{\Delta v}\left(\sqrt{\frac{2 \tau_{\sigma}}{n_{\sigma}}}-\sqrt{-2 \epsilon_{I \sigma}}\right),
$$

which goes to zero asymptotically.

\section{PERFORMANCE OF THE REGULAR BECKE-JOHNSON TERM IN AN EXTERNAL ELECTRICAL FIELD}

We have implemented Slater exchange plus the correction of Eq. (12) in an electronic structure code (cf. the Appendix for details). It is an exchange potential functional (rather than energy functional, as is common for semi-local DFT). However, we do not have an exchange energy functional whose derivative gives this potential. One can nevertheless perform polarizability calculations without a total energy, by iterating the Kohn-Sham scheme for potential selfconsistency and computing the polarizability from the dipole moment. ${ }^{26}$

The potential functional is tested in a common test model for benchmarks of electric polarization, the hydrogen chain. ${ }^{7}$ It consists of a number of hydrogen atom pairs placed equidistantly on a straight line in a three-dimensional volume. The intrapair distance is chosen to be 2 bohr, with a distance between the pairs of $3 \mathrm{bohr}$. This model is used in the present work for exactly the same reasons, as stated in Ref. 12: (i) the model mimics the electronic features of polymers like polyacetylene such as bond-length alternation, high and directional electron mobility, and large response coefficients, while at the same time their electronic structure is transparent enough to keep technical details (basis sets or grid parameters) well controlled; (ii) $a b$ initio correlated wave function calculations are available for comparison; ${ }^{27}$ (iii) the current-DFT calculations of Ref. 28 showed that among various molecular chains, hydrogen chains are the ones that are the most difficult to describe accurately within DFT. It can thus be expected that if they are described correctly, then other chains will be described correctly too.

However, using Slater exchange plus the BJ correction as a potential functional does not give impressive test results for hydrogen chains in electrical fields. Figure 3 shows the difference between the exchange potential of a hydrogen chain in a weak electric field, and no external field, $v_{\mathrm{x} \sigma}^{F \neq 0}-v_{\mathrm{x} \sigma}^{F=0}$. This difference is the typical way of looking at hydrogen chain results ${ }^{7,8,12}$ since it gives a clear view of the effect of introducing the external electrical field. The plots show how 

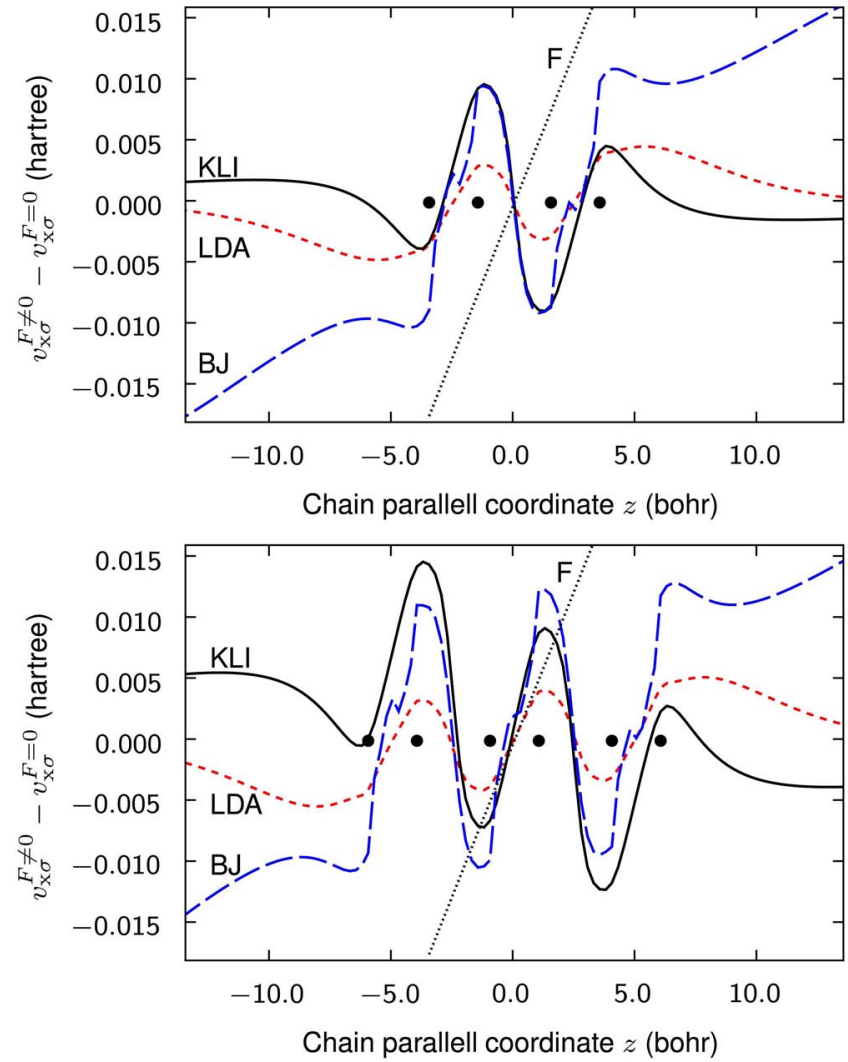

FIG. 3. (Color online) The difference between the exchange potential for calculations with and without an external electrical field of strength $F=0.005$ hartree/bohr (shown in the figure). The upper figure is for $\mathrm{a}_{4}$ chain, and the lower for a $\mathrm{H}_{6}$ chain (atom positions are indicated by circles). The figure shows that calculations using the BJ correction term are not very similar to the exact exchange as approximated by KLI nor does it behave properly far outside the system.

the potential difference of the methods presented here does not resemble the result of the exact exchange (as approximated by KLI) and has severe issues at the asymptotics. The BJ correction leads to a potential that structurewise seems to be an improvement over regular LDA, but it is "tilted" with respect to the KLI curves.

For the calculations without any external field, there is no apparent problem with the BJ correction term. We find the performance to be roughly as good as for regular atoms (as was investigated in the original BJ work ${ }^{17}$ ). The tilt in the difference plots comes solely from the calculation of the system with an external field. Far outside the system, the tilt becomes very linear in $z$, as is shown in Fig. 4. In the following, we investigate the origin of this tilt.

\section{ASYMPTOTICS OF THE BECKE-JOHNSON TERM IN AN EXTERNAL ELECTRICAL FIELD}

The issues with the BJ correction term asymptotics can be understood by revisiting the one-dimensional model above but now for a system with a weak external electrical field $v_{\text {ext }}(z)=F z$. Outside the system, the Kohn-Sham potential from the atomic potentials falls off as $1 / z$, and the potential

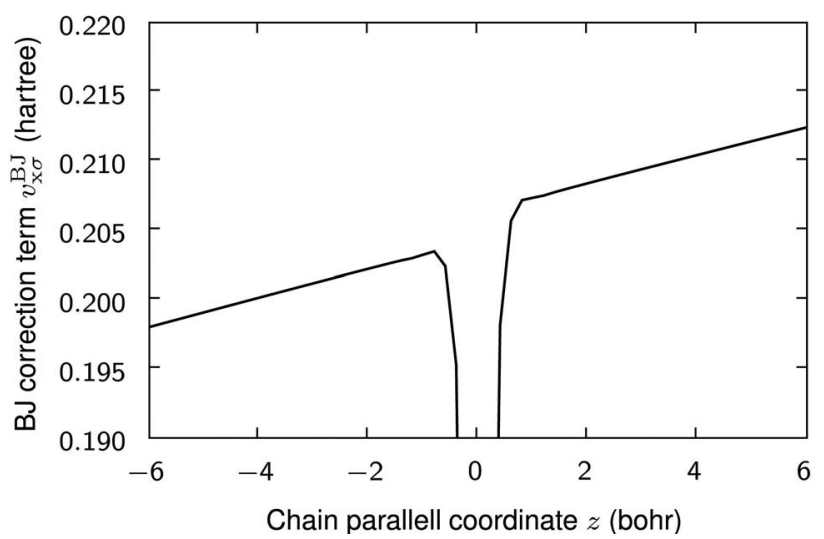

FIG. 4. The BJ correction term $v_{\mathrm{x} \sigma}^{\mathrm{BJ}}$ of Eq. (8) for a single hydrogen atom in an electrical field $F=0.005$ hartree/bohr. The BJ correction term is here calculated from self-consistent KLI KS orbitals (the other figures show Slater exchange + BJ correction done fully self-consistently). The figure shows how the BJ term slopes linearly outside a system in an electrical field.

is dominated by the linear electrical field term. In this case, the KS orbital eigenequation approaches the following form:

$$
-\frac{1}{2} \frac{d^{2}}{d z^{2}} \phi(z)+F z \phi(z)=\epsilon \phi(z) .
$$

The transformation $\eta=(2 F)^{1 / 3}(z-\epsilon / F)$ turns this into an Airy differential equation ${ }^{29} \frac{d^{2}}{d \eta^{2}} \phi(\eta)=\eta \phi(\eta)$ with the general solution

$$
\phi(\eta)=A_{1} \operatorname{Ai}(\eta)+A_{2} \operatorname{Bi}(\eta)
$$

Hence, for a system in an infinite external electrical field, all the KS orbitals approach this form asymptotically for large positive and negative values of $z$. Technically, a given orbital can have different values of the constants $A_{1}$ and $A_{2}$ in the two limits since there is an unspecified structure present in between these limits. However, $\operatorname{Bi}(\eta)$ diverges for large positive arguments, and in the discussion below, we will use this to argue that sane boundary conditions require $A_{2}$ to be effectively zero in both limits.

For negative values of $z$ large enough to make the argument to the Airy functions negative, $\eta<0$, the orbitals in Eq. (14) become unbound and oscillatory. This is a well known issue for a system in an infinite electrical field: formally, the states are unbound. In practice, this is not a problem when (i) the field is weak enough, (ii) the physical realization of the system is within a somewhat limited volume, and (iii) the highest occupied orbital is sufficiently strongly bound. Should any one of these related assumptions not hold true, the system would undergo significant ionization, which is a situation we are not concerned with here.

In other words, since the strength of the field, $v_{\text {ext }}(z)$, grows linearly as $z \rightarrow-\infty$, we cannot consider a truly infinite system. The system size has to be limited so that the weakest bound orbital (i.e., the highest occupied one) is still strongly bound in comparison with the ionization strength of the field, i.e., 


$$
\epsilon_{I \sigma} \ll F z
$$

Exactly the same inequality also falls out directly from the form of the orbitals in Eq. (14). The Airy functions turn oscillatory for $\eta<0$. To well avoid this region requires $\eta$ $\gg 0$, which is equivalent to Eq. (15).

The limited size of the system can be manifested in an actual finite field calculation through boundary conditions. The actual choice of boundary should not be important, but it makes sense to use a symmetric "box" condition and thus force the orbitals to 0 at some $z= \pm z_{\max }$, where $z_{\max } \ll$ $-\epsilon_{I \sigma} / F$. We must thus assume that such a $z_{\max }$ exists that yet is large enough for the boundaries not to interfere with the structure of the system, i.e., $z_{\text {struct }} \ll z_{\text {max }}$. This is a natural assumption for an "infinitely large, weak field" situation because if the boundaries of the external field interfere with the structure of the system, the field will certainly not appear to be "infinitely large."

What behavior is expected from the occupied KS orbitals in the infinitely large, weak field we are trying to model? Outside the part of the system with structure, i.e., $|z|$ $>z_{\text {struct }}$, they should decay exponentially, similar to a situation without field. Hence, for large values of $|z|$, the orbitals should be essentially zero. Given the extreme exponential divergence of the Airy $\mathrm{Bi}$ function for positive arguments, this can only be achieved if its coefficient $A_{2}$ in Eq. (14) also is essentially zero. More formally, the further away the boundary of the system can be placed without the field ionizing the system, i.e., the larger $-\epsilon_{I \sigma} / F$ is, the smaller $A_{2}$ will have to be in relation to $A_{1}$ in Eq. (14).

In the hydrogen chain calculations of the present work where $F=0.005$ hartree/bohr, a typical value of the energy of the highest occupied orbital can be $\epsilon_{I \sigma} \approx-0.5$ hartree, giving that $z_{\max } \ll 100 \mathrm{bohr}$, whereas $z_{\text {struct }} \approx 10$. If a boundary is placed at $z_{\max }=25$, the corresponding arguments for the Airy functions at the left and right boundaries are $\eta_{\text {left }} \approx 16.2$ and $\eta_{\text {right }} \approx 26.9$. The boundary condition would require the orbital in Eq. (14) to be zero for these arguments. Hence, the least strict bound on $A_{2}$ is at the left boundary and is given in the current example by $\left|A_{2}\right| \approx 10^{-38}\left|A_{1}\right|$.

Furthermore, since we have already concluded that the argument $\eta$ to the Airy functions in the orbitals of Eq. (14) is always positive and large, the Airy Ai function is well represented by its large argument first order asymptotic throughout the whole system (i.e., for both positive and negative $z$ ),

$$
\operatorname{Ai}(\eta) \rightarrow \frac{1}{2} \frac{1}{\sqrt{\pi \sqrt{\eta}}} e^{-2 \eta^{3 / 2} / 3}
$$

The conclusion of the above analysis of our "infinitely large, weak field model" is that the occupied KS orbitals in such a system have the following asymptotic form:

$$
\phi_{i \sigma}(z) \rightarrow \sim \eta^{-1 / 4} e^{-2 \eta^{3 / 2} / 3}, \quad \eta=(2 F)^{1 / 3}\left(z-\frac{\epsilon_{i \sigma}}{F}\right) .
$$

Similar to the zero field situation leading up to Eq. (10), the largest occupied orbital dominates the asymptotic density and kinetic energy density, and all other terms in their orbital sums can be left out. In the current situation, the asymptotic of the BJ term becomes

$$
v_{\mathrm{x} \sigma}^{\mathrm{BJ}} \rightarrow C_{\Delta v}\left|\frac{\nabla \phi_{I \sigma}(z)}{\phi_{I \sigma}(z)}\right|=C_{\Delta v} \sqrt{2\left(F z-\epsilon_{I \sigma}\right)} .
$$

This properly reduces to Eq. (10) for the zero field situation $F=0$. The expression can be simplified one step further by realizing that under the assumptions we have made for the model, the expression will predominantly look linear in $z$. This can be realized by a straightforward expansion in $z$ for $|F z| \ll\left(-\epsilon_{I \sigma}\right)$, giving

$$
v_{\mathrm{x} \sigma}^{\mathrm{BJ}} \rightarrow C_{\Delta v} \sqrt{-2 \epsilon_{I \sigma}}+C_{\Delta v} \frac{F}{\sqrt{-2 \epsilon_{I \sigma}}} z .
$$

Hence, the final conclusion from our analysis of the onedimensional model above is that the total correction, Eq. (12), should also include the linear term from the external electrical field, giving

$$
v_{\mathrm{x} \sigma}^{\mathrm{corr}}=C_{\Delta v}\left(\sqrt{\frac{2 \tau_{\sigma}}{n_{\sigma}}}-\sqrt{-2 \epsilon_{I \sigma}}-\frac{F}{\sqrt{-2 \epsilon_{I \sigma}}} z\right),
$$

which is used in the final total exchange potential

$$
v_{\mathrm{x}}=v_{\mathrm{x} \sigma}^{\text {Slater }}+v_{\mathrm{x} \sigma}^{\text {corr }} \text {. }
$$

\section{APPLICATION OF THE CORRECTED EXCHANGE POTENTIAL FUNCTIONAL}

The above analysis has resulted in an exchange potential functional that includes a number of quantities normally not associated with semi-local DFT: the Slater potential $v_{\mathrm{x}}^{\text {Slater }}$, the external field $F$, and the energy of the highest occupied eigenvalue $\epsilon_{I \sigma}$. Also, formally, the kinetic energy density $\tau$ is semi-local in the KS orbitals rather than the electron density (but this does not impose any significant computational overhead, and it is an ingredient in several so-called meta-GGA functionals). It is, however, still entirely feasible to implement the functional with these quantities. The point remains that the expression that actually reproduces the necessary features of exact exchange is the peculiar $\sqrt{2 \tau_{\sigma} / n_{\sigma}}$ construction, which is composed by accepted semi-local quantities. That said, it is entirely possible that the non-semi-local quantities in Eq. (21) can be eliminated. For example, BJ already proposed using the Becke-Roussel approximation in place of the Slater potential. ${ }^{30}$

We have implemented the expression in Eq. (21), and the results are shown in Fig. 5 and Table I. The figure shows that the tilt of the potential observed for the uncorrected BJ term in Fig. 3 is now gone. The term linear in $z$ in Eq. (20) precisely counteracts the tilt. The result is an exchange potential surprisingly close to the one obtained from KLI. Furthermore, Table I shows the polarizability results to be of the same accuracy as the ones from exact exchange methods.

It is common to discuss the performance of functionals for electrical field calculations in terms of the so-called "field counteracting term." The point is that the exact exchange potential includes a slope that counteracts the external elec- 

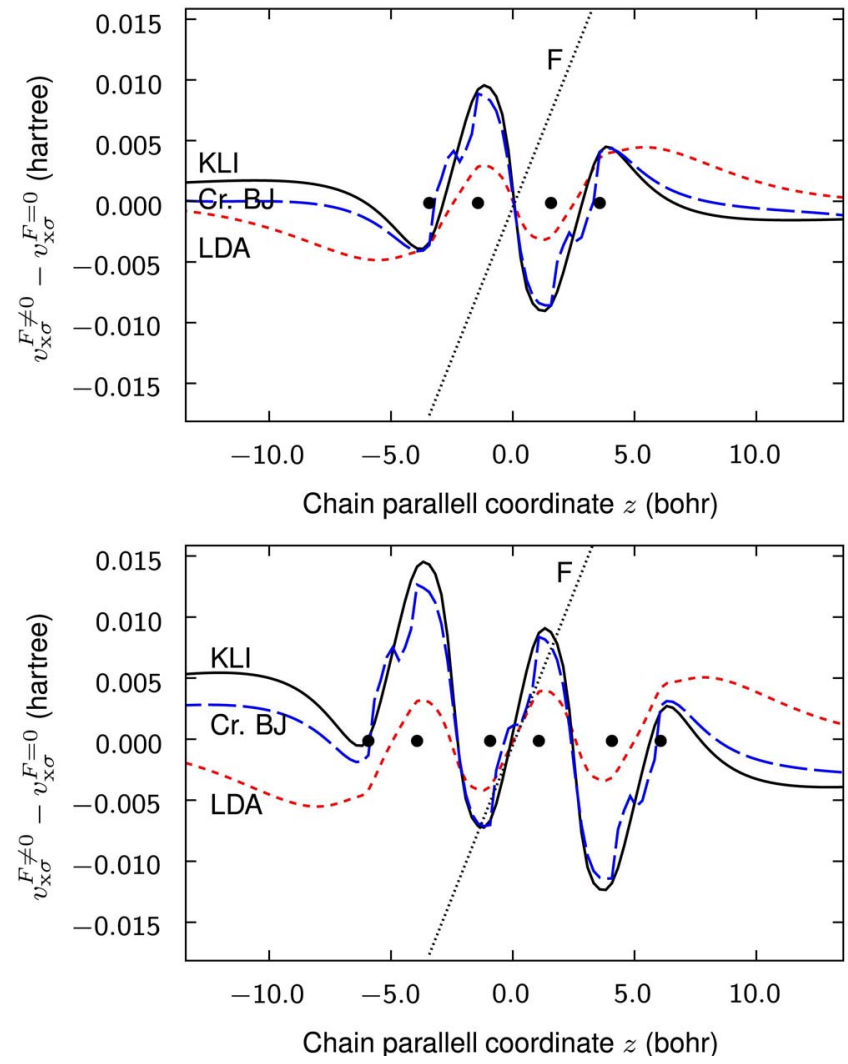

FIG. 5. (Color online) As in Fig. 3, the difference between the exchange potential for calculations with and without an external electrical field of strength $F=0.005$ hartree/bohr for a $\mathrm{H}_{4}$ and $\mathrm{H}_{6}$ chain. However, now, the exchange functional used has been further corrected with the additional linear term derived in Eq. (20), and the similarity with exact exchange (as approximated by KLI) is apparent.

trical field. This slope is reproduced by both KLI and the corrected BJ term potential, as is clearly seen in Fig. 5. In contrast, the LDA plot appears to have a weak sloping in the wrong "with-field" direction. This behavior is typical for commonly used semi-local functionals.

Equation (20) contains a term that explicitly depends on the external field $F$ and $z$ position, and it might be tempting to dismiss this term as an artificial "trick" to introduce a field counteracting term that was missing in the original BJ results. However, such a trick could not be performed for regular semi-local functionals such as LDA. The reason is that they already decay properly to zero outside the hydrogen chain. It is just because the asymptotics for the uncorrected BJ term in Fig. 3 are behaving improperly that it is possible to introduce a term that counteracts the incorrect tilt and changes the asymptotics. Hence, it makes more sense to interpret Eq. (20) as the removal of an incorrect field term that is present within the original $\mathrm{BJ}$ term when performing calculations in an external electrical field-rather than as the addition of an artificial field counteracting term.

In a more generalized view, potential functionals should normally decay to zero in the infinite boundary. The original BJ correction term did not fulfil this when used in a system with an external electrical field. The correction in Eq. (20) handles this problem.
TABLE I. Numerical polarizabilites in bohr ${ }^{3}$ of DFT using Eq. (21) compared to other methods: local density approximation (LDA), the KLI approximation to exact exchange (x-KLI), full exact exchange (x-OEP) from Ref. 12, Hartree-Fock (HF), and Møller-Plesset (MP4) from Refs. 7, 31, and 32. The large overestimation of LDA is typical for DFT with semi-local functionals. The HF and MP4 methods are generally considered to reproduce polarizabilites well.

\begin{tabular}{lcc}
\hline \hline & $\mathrm{H}_{4}$ & $\mathrm{H}_{6}$ \\
\hline DFT, Eq. (21) & 30.1 & 54.9 \\
DFT, LDA & 37.7 & 73.3 \\
x-KLI & 33.2 & 60.4 \\
x-OEP & 32.2 & 56.6 \\
HF & 32.0 & 56.4 \\
MP4 & 29.5 & 51.6 \\
\hline \hline
\end{tabular}

\section{FUTURE GENERALIZATION INTO A FULL SEMI-LOCAL EXCHANGE CORRELATION FUNCTIONAL}

The ultimate goal of the work started in this paper is a good description of both structural and electrical properties of molecular chains. This can only be reached with a suitable description of both exchange and correlation. Now that a suitable exchange functional is available, the next step should be to create a correlation functional that works well together with this exchange functional. Even though exchange is handled as an exchange potential functional, there is nothing that prevents combining it with an ordinary semilocal correlation energy approximation where the correlation potential is given via the usual functional derivative. There exists a wealth of such expressions in the literature. However, since the exchange potential functional presented here focuses heavily on the derivative discontinuity and step structure found in atoms and molecules, it would make sense to start this development from an expression that incorporates the physics of bounded systems. Such an expression can be constructed either from an empirical fit to such systems, e.g., by fitting to data for atoms, as is done in many semiempirical functionals; alternatively, it can be based on a fit to bounded nonempirical systems, similar as the AM05 correlation functional was constructed in a recent paper involving one of the present authors. ${ }^{33}$

\section{CONCLUSIONS}

In conclusion, in this paper, we have (i) shown that it is possible to use semi-local quantities to reproduce a step structure and derivative discontinuity in the exchange potential that behaves correctly for fractional particle numbers, something that has traditionally been associated with nonlocal exchange methods; (ii) produced an exchange potential functional incorporating this treatment; (iii) implemented the functional in a density functional code suitable for polarization calculations; (iv) performed calculations that show the functional to be as accurate as exact exchange methods for the polarizability of hydrogen chains; (v) discussed how fu- 
ture work may combine this exchange potential functional with a suitable correlation treatment. Thus, we have shown that the response of hydrogen chains, which has been classified as "ultra non-local," 7,8 can be described by a density functional that employs the Kohn-Sham orbitals only in a semi-local way.

\section{ACKNOWLEDGMENTS}

We thank Kieron Burke for helpful comments regarding the relation between the energy and our potential functional. We gratefully acknowledge support from the Alexander von Humboldt Foundation.

\section{APPENDIX: DETAILS OF CALCULATIONS}

The Mg ion calculations presented in Figs. 1 and 2 were performed using an atomic DFT code with its origins tracing back to an early work by Talman and Shadwick ${ }^{34}$ (but which has been heavily modified over the years by a number of people). Fully self-consistent spin-polarized DFT was used on a one-dimensional logarithmic grid of 800 mesh points $r \in\left\{e^{-8+0.015 i}\right\}_{i=0}^{799}$. The orbitals in Fig. 1 were filled successively alternating between spin up and spin down.

For the spin-polarized calculations in Figs. 3-5, and Table I, we used the same methods as described in Ref. 26, using a heavily modified version of the publicly available electronic structure program PARSEC ${ }^{35}$ with a functional independent hydrogen pseudopotential. Specifically, fully selfconsistent spin-polarized DFT calculations were performed on a real-space grid with a grid spacing of $0.25 \mathrm{bohr}$ and with an energy convergence criterion for the KS iterations of $0.5 \times 10^{-7}$ hartree. No relaxation was performed (the hydrogen chain benchmark numbers are for hydrogen atoms at fixed positions). The converged grid boundaries were for the $\mathrm{H}_{4}$ chain, an ellipsoidic grid within the boundaries $x \in[-10,10], y \in[-10,10], z \in[-20,20]$ (in bohr), and for the $\mathrm{H}_{6}$ chain, an ellipsoidic grid within the boundaries $x \in[-10,10], y \in[-10,10], z \in[-30,30]$.
${ }^{1}$ D. R. Kanis, M. A. Ratner, and T. J. Marks, Chem. Rev. (Washington, D.C.) 94, 195 (1994).

${ }^{2}$ P. Hohenberg and W. Kohn, Phys. Rev. 136, B864 (1964).

${ }^{3}$ W. Kohn and L. J. Sham, Phys. Rev. 140, A1133 (1965).

${ }^{4}$ J. P. Perdew, in Electronic Structure of Solids 91, edited by P. Ziesche and H. Eschrig (Akademic, Berlin, 1991), p. 11; J. P. Perdew, J. A. Chevary, S. H. Vosko, K. A. Jackson, M. R. Pederson, D. J. Singh, and C. Fiolhais, Phys. Rev. B 46, 6671 (1992).

${ }^{5}$ J. P. Perdew, K. Burke, and M. Ernzerhof, Phys. Rev. Lett. 77, 3865 (1996).

${ }^{6}$ B. Champagne, E. A. Perpéte, S. J. A. van Gisbergen, E.-J. Baerends, J. G. Snijders, C. Soubra-Ghaoui, K. A. Robins, and B. Kirtman, J. Chem. Phys. 109, 10489 (1998).

${ }^{7}$ S. J. A. van Gisbergen, P. R. T. Schipper, O. V. Gritsenko, E. J. Baerends, J. G. Snijders, B. Champagne, and B. Kirtman, Phys. Rev. Lett. 83, 694 (1999).

${ }^{8}$ O. V. Gritsenko, S. J. A. van Gisbergen, P. R. T. Schipper, and E. J. Baerends, Phys. Rev. A 62, 012507 (2000).

${ }^{9}$ N. T. Maitra and M. van Faassen, J. Chem. Phys. 126, 191106 (2007)

${ }^{10}$ P. Mori-Sánchez, Q. Wu, and W. Yang, J. Chem. Phys. 119, 11001 (2003).

${ }^{11}$ H. Iikura, T. Tsuneda, T. Yanai, and K. Hirao, J. Chem. Phys. 115, 3540 (2001).

${ }^{12}$ S. Kümmel, L. Kronik, and J. P. Perdew, Phys. Rev. Lett. 93, 213002 (2004).

${ }^{13}$ H. Sekino, Y. Maeda, and M. Kamiya, Mol. Phys. 103, 2183 (2005).

${ }^{14}$ T. Körzdörfer, M. Mundt, and S. Kümmel, arXiv:0708.2870, Phys. Rev. Lett. (to be published).

${ }^{15}$ C. D. Pemmaraju, S. Sanvito, and K. Burke, Phys. Rev. B 77, 121204 (2008).

${ }^{16}$ A. Ruzsinszky, J. P. Perdew, G. I. Csonka, G. E. Scuseria, and O. A. Vydrov, report (unpublished).

${ }^{17}$ A. D. Becke and E. R. Johnson, J. Chem. Phys. 124, 221101
(2006).

${ }^{18}$ J. P. Perdew, R. G. Parr, M. Levy, and J. L. Balduz, Jr., Phys. Rev. Lett. 49, 1691 (1982).

${ }^{19}$ J. B. Krieger, Yan Li, and G. J. Iafrate, Phys. Lett. A 146, 256 (1990).

${ }^{20}$ J. B. Krieger, Yan Li, and G. J. Iafrate, Phys. Rev. A 45, 101 (1992).

${ }^{21}$ S. Kümmel and L. Kronik, Rev. Mod. Phys. 80, 3 (2008).

${ }^{22}$ R. van Leeuwen, O. Gritsenko, and E. J. Baerends, Z. Phys. D: At., Mol. Clusters 33, 229 (1995).

${ }^{23}$ M. Lein and S. Kümmel, Phys. Rev. Lett. 94, 143003 (2005).

${ }^{24}$ M. Mundt and S. Kümmel, Phys. Rev. Lett. 95, 203004 (2005).

${ }^{25}$ A discussion of the asymptotic behavior Kohn-Sham orbitals can be found, e. g., in T. Kreibich, S. Kurth, T. Grabo, and E. K. U. Gross, Adv. Quantum Chem. 33, 31 (1999).

${ }^{26}$ S. Kümmel and L. Kronik, Comput. Mater. Sci. 35, 321 (2006).

${ }^{27}$ B. Champagne, D. H. Mosley, M. Vračko, and J.-M. André, Phys. Rev. A 52, 178 (1995).

${ }^{28}$ M. van Faassen, P. L. de Boeij, R. van Leeuwen, J. A. Berger, and J. G. Snijders, Phys. Rev. Lett. 88, 186401 (2002).

${ }^{29}$ Handbook of Mathematical Functions, edited by M. Abramowitz and I. A. Stegun (Dover, New York, 1970).

${ }^{30}$ A. D. Becke and M. R. Roussel, Phys. Rev. A 39, 3761 (1989).

${ }^{31}$ M. Grüning, O. V. Gritsenko, and E. J. Baerends, J. Chem. Phys. 116, 6435 (2002).

${ }^{32}$ S. Kümmel, J. Comput. Phys. 201, 333 (2004).

${ }^{33}$ R. Armiento and A. E. Mattsson, Phys. Rev. B 72, 085108 (2005).

${ }^{34}$ J. D. Talman and W. F. Shadwick, Phys. Rev. A 14, 36 (1976).

${ }^{35}$ L. Kronik, A. Makmal, M. L. Tiago, M. M. G. Alemany, M. Jain, X. Huang, Y. Saad, and J. R. Chelikowsky, Phys. Status Solidi B 243, 1063 (2006); http://www.ices.utexas.edu/parsec/index.html

${ }^{36}$ The total energy $E=T_{s}+J+V+E_{\mathrm{xc}}$, where $J$ is the internal energy of a classic repulsive gas, or "Hartree energy," and $V$ is the external potential energy. The derivative with respect to particle number of $J$ and $V$ is continuous due to their simple dependence on the density. 